\title{
La pratica infermieristica basata sui modelli teorici: uno studio qualitativo sulla percezione degli Infermieri
}

\author{
Nursing practice based on theoretical models: a qualitative study of nurses' perception
}

\author{
Giovanna Amaducci1 Marina lemmi ${ }^{2} \quad$ Marzia Prandi3 ${ }^{3}$ Angelina Saffioti 3
}

Marika Carpanoni5 Daniela Mecugni6

\begin{abstract}
RIASSUNTO
Molti studiosi sostengono che teoria e teorizzazione sono strettamente correlate alla pratica clinica e che il sapere disciplinare non procede solo dall'applicazione dei principi generali di grandi teorie ai casi particolari, ma si sviluppa, in maniera più pertinente, a partire dallo specifico contesto assistenziale nel quale si realizza. Ogni infermiere, infatti, possiede un modello mentale del quale può o meno essere consapevole, che motiva e sostanzia ogni azione e scelta professionale.

Il presente studio descrive il modello teorico infermieristico che orienta l'agire professionale, il modello mentale e le conoscenze tacite ad esso sottese. Identifica nel modello teorico esplicito del gruppo professionale che gli infermieri partecipanti rappresentano, aspetti di continuità con il modello teorico proposto da questo Corso di Laurea in Infermieristica,

Sono stati realizzati 4 focus group ai quali hanno partecipato complessivamente 22 Infermieri, rappresentanti di quasi tutte le unità operativa dell'Arcispedale Santa Maria Nuova di Reggio Emilia.

E' possibile sostenere che il modello teorico infermieristico di ciascun gruppo professionale è il risultato di conoscenze tacite, che contribuiscono a definire il modello mentale di ciascun professionista e di un modello teorico esplicito cui sono sottesi contenuti teorici appresi applicati consapevolmente e riadattati alla/dalla pratica assistenziale. Ragionare sull'uso della teoria nella pratica ha permesso di dare visibilità a un modello teorico esplicito autenticamente infermieristico orientato ai bisogni della persona, nella sua complessità in specifici contesti.

Parole chiave: modelli teorici dell'infermieristica, conoscenze tacite, sapere esplicito, pratica clinica, Focus Group.
\end{abstract}

\section{ABSTRACT}

Many faculty argue that theory and theorizing are closely related to the clinical practice, that the disciplinary knowledge grows, more relevantly, from the specific care context in which it takes place and, moreover, that knowledge does not proceed only by the application of general principles of the grand theories to specific cases. Every nurse, in fact, have a mental model, of what may or may not be aware, that motivate and substantiate every action and choice of career. The study describes what the nursing theoretical model is; the mental model and the tacit knowledge underlying it. It identifies the explicit theoretical model of the professional group that rapresents nursing partecipants, aspects of continuity with the theoretical model proposed by this degree course in Nursing..

Methods Four focus groups were made which were attended by a total of 22 nurses, rapresentatives of almost every Unit of Reggio Emilia Hospital's.

We argue that the theoretical nursing model of each professional group is the result of tacit knowledge, which help to define the personal mental model, and the theoretical model, which explicitly underlying theoretical content learned applied consciously and reverted to / from nursing practice.

Reasoning on the use of theory in practice has allowed us to give visibility to a theoretical model explicitly nursing authentically oriented to the needs of the person, in all its complexity in specific contexts.

Key Words: nursing theories, tacit knowledge, explicit knowledge, clinical practice, focus group

1 Tutor-Docente Infermiere, Corso di Laurea in Infermieristica - Sede di Reggio Emilia Università degli Studi di Modena e Reggio Emilia Corrispondenza: Amaducci.giovanna@asmn.re.it

2 Direttore della Direzione delle Professioni Sanitarie, Arcispedale Santa Maria Nuova di Reggio Emilia

3 Responsabile Formazione e Sviluppo della Direzione Professioni Sanitarie, Arcispedale Santa Maria Nuova di Reggio Emilia

4 Coordinatore Infermieristico Residenza Sanitaria Riabilitativa di Albinea - ASL Reggio Emilia

5 Tutor Infermiere, Corso di Laurea in Infermieristica - Sede di Reggio Emilia, Università degli Studi di Modena e Reggio Emilia

6 Ricercatore MED/45 - Scienze Infermieristiche Generali, Cliniche e Pediatriche, Coordinatore Corso di Laurea in Infermieristica -sede di Reggio Emilia Università degli Studi di Modena e Reggio Emilia

\section{INTRODUZIONE}

T a teoria è un costrutto sistematico, fondato scientificamente e logicamente correlato a un insieme di concetti che identificano le componenti essenziali della pratica infermieristica insieme con le basi teoriche e i valori richiesti per l'agire professionale (Fawcett, 1992; Im et al., 1999; Manara, 2002b; Butts et al., 2012).

Per teoria si intende quindi una "rappresentazione concettuale" che permette di descrivere, spiegare e prevenire, o risolvere, il problema oggetto di studio 
(Im et al., 1999; Manara, 2002b).

L'agire professionale è impossibile senza un "concetto astratto della professione", un'immagine mentale (Alborghetti, 1997), in grado di definire e caratterizzare l'ottica attraverso la quale mettere a fuoco l'oggetto specifico di conoscenza (Calamandrei, 2003).

In ambito infermieristico, le teorie descrivono l'oggetto di studio della disciplina, ne identificano lo scopo specifico, parlano del metodo e delle sue applicazioni. L'interpretazione di alcuni concetti, quali persona, salute/malattia, ambiente ed assistenza infermieristica vengono assunti come riferimenti filosofici-valoriali che delimitano il campo di elaborazione del pensiero infermieristico (Fawcett, 1992; Im et al., 1999; Calamandrei, 2003; McCrae, 2012).

La letteratura (Reilly, 1975; Daly et al., 1999; Manara, 2002a) riporta che ogni situazione assistenziale acquista un senso ben preciso alla luce di riferimenti teorici e modelli concettuali che ne organizzano la finalità e danno ragione delle singole azioni. Ogni infermiere possiede immagini mentali, delle quali può o meno esserne consapevole (Manara, 2002b). Si tratta di modelli interpretativi costruiti sulla base, da un lato, dell'esperienza accumulata, dall'altro, delle norme e dei valori morali-etico-deontologici che orientano l'agire professionale. Questi modelli interpretativi agiscono come "filtri" che inducono il professionista a selezionare una porzione di ambiente rilevante, attribuire significato ai fatti, scegliere il corso delle azioni più opportune dando forma e significato alla realtà che lo circonda (Fealy, 1999).

Raramente, però, il clinico è stato ritenuto il produttore di conoscenze scientifiche - ambito proprio e specifico di colui che pensa - più spesso è st $\&$ Meleisato considerato colui che applica teorie o sottopone alla prova dei fatti le conoscenze da altri concepite, o colui che fornisce idee o problemi ricercabili. Questo tradizionale modello di concepire lo sviluppo teorico della disciplina infermieristica, ha determinato un progressivo distanziamento fra infermieri che fanno ed altri che pensano, a tale punto che l'1\% degli infermieri producono sapere per il restante 99\%: quasi come se gli infermieri che fanno non pensassero e quelli che pensano non facessero (Fawcett, 1999; Reed, 2008).

L'attuale esigenza di sviluppo professionale richiede, invece, di comprendere se e come gli infermieri producono sapere anche attraverso il loro agire, accrescendo in tale modo la convergenza fra coloro che descrivono l'assistenza come dovrebbe essere e chi la rappresenta per ciò che è (De Marinis et al., 2004a).

Le teorie infermieristiche studiate a scuola sono spesso abbandonate nella clinica e c'è chi si chiede se esistano ancora o siano estinte (Tierney, 1998), tanto è stato minimo il loro impatto sulla pratica assistenziale (Cody, 1994), la loro reale utilità per la pratica (Luker, 1988;
Kenny, 1993;) e per lo sviluppo teorico-concettuale della stessa disciplina Infermieristica (McCrae, 2012).

In Italia, la diffusione di un pensiero teorico largamente accettato e la sua applicazione nella pratica assistenziale, sembrano costituire obiettivi ancora deboli della comunità infermieristica (De Marinis et al., 2004a), infatti se si chiede agli infermieri italiani quali modelli ispirano il loro lavoro quotidiano, alcuni risponderanno di non averne, altri riporteranno vaghi ricordi su alcuni concetti teorici (nursing, bisogno, olismo....) richiamati dalle esperienze scolastiche (Degan, 2001).

Si sta diffondendo molta letteratura (Im et al., 1999; Reed et al., 2008) a sostegno del fatto che il sapere disciplinare si sviluppi, in maniera più pertinente, a partire dallo specifico contesto assistenziale nel quale si realizza e non proceda solo dall'applicazione dei principi generali, di grandi teorie e modelli concettuali, ai casi particolari (metodo deduttivo). Per questo, alcuni autori (Im et al., 1999; Degan, 2001; Marrs et al., 2006) prospettano, il superamento del 'mito della grande teoria unificatrice' ed aprono alla possibilità di illuminare l'agire assistenziale attraverso teorie elaborate dalla pratica stessa, cioè costruite nei contesti clinici, "al letto del paziente"; a tale proposito Reed et al. (2008) sostiene che lo sviluppo del sapere teorico debba derivare dalla pratica assistenziale.

La ricerca riportata, si inserisce in un progetto più ampio, che vede la collaborazione del Corso di Laurea in Infermieristica, Sede di Reggio Emilia, con la Direzione delle Professioni Sanitarie dell'ospedale cittadino, dal titolo "Analisi del processo di identificazione dei problemi della persona assistita". La finalità di tale progetto, è quella di introdurre, in alcune unità operative, la progettazione assistenziale e l'applicazione del processo di nursing attraverso l'utilizzo della Tassonomia NANDA-NOC-NIC.

Il Processo, scollegato da una guida teorica, è un "movimento nel buio" capace di disperdere le coordinate di ogni relazione di cura e di lasciare l'assistenza infermieristica nel terreno inamovibile della subordinazione tecnica (Aggleton et al., 1987; Calamadrei, 2003).

\section{Scopo dello studio}

Descrivere il modello teorico infermieristico al quale fanno riferimento i gruppi infermieristici dell'Arcispedale Santa Maria Nuova di Reggio Emilia.

In particolare, lo studio intende:

- mettere in luce il significato di modello teorico dell'infermieristica;

- descrivere il modello mentale e le conoscenze tacite ad esso sottese;

- identificare nel modello teorico esplicito, aspetti di continuità con il modello teorico dei bisogni umani di Virginia Henderson, modificato secondo Adam e Bizier (Motta, 2002) proposto dal Corso di Laurea in Infermieristica. 


\section{MATERIALI E METODI}

\section{Tipo di studio}

Studio qualitativo con utilizzo del focus group su campione non probabilistico. La scelta di utilizzare il focus group, piuttosto che l'intervista, è sostanziato dalla letteratura (Baldry, 2005) che considera tale metodologia, proprio perché favorisce l'interazione fra i componenti del gruppo, particolarmente adeguata alla genesi di evocazioni, associazioni, richiami.

\section{Il campione}

Ogni focus groups ha coinvolto 6-10 Infermieri. Questa numerosità dei partecipanti ai focus, definito numero critico (Baldry, 2005), ha inteso garantire la più ampia e ricca condivisione/circolazione dei diversi punti di vista nel tempo disponibile (De Marinis et al., 2004b).

\begin{tabular}{|c|c|}
\hline $\begin{array}{c}\text { Caratteristiche demografiche e } \\
\text { lavorative }\end{array}$ & Numero assoluto \\
\hline Età (media $\pm d s)$ & $41 \pm 8$ \\
\hline \multicolumn{2}{|l|}{ Sesso } \\
\hline Maschi & 2 \\
\hline Femmine & 20 \\
\hline Totale infermieri & 22 \\
\hline Anni di Lavoro (media $\pm d s)$ & $9 \pm 4$ \\
\hline \multicolumn{2}{|l|}{ Situazione Lavorativa - } \\
\hline Tempo Pieno & 19 \\
\hline Part-Time $75 \%$ & 3 \\
\hline
\end{tabular}
preso parte ai focus groups.
I criteri di selezione utilizzati sono stati i seguenti: 1-2 Infermieri Guida per ciascuna Unità Operativa dei 7 dipartimenti dell'Azienda Ospedaliera Santa Maria Nuova di Reggio Emilia (Tabella I-II), almeno 3 anni di anzianità, selezionati dal Coordinatore in base alla turistica e alla disponibilità a partecipare al progetto. Sono stati scelti Infermieri Guida perché considerati più abituati a termini, magari non utilizzati nella pratica quotidiana, quali modelli teorici o modelli concettuali, proprio grazie al confronto con gli studenti.

$\mathrm{Ha}$ rappresentato criterio di esclusione: l'essere Coordinatore e/o Dirigente Infermieristico di Dipartimento, per evitare condizionamenti sui componenti del gruppo.

Complessivamente sono stati identificati 27 partecipanti.

\section{Modalità di realizzazione}

Sono stati allestiti 4 focus groups della durata di circa 120 minuti (De Marinis et al., 2004b). In due focus gli infermieri appartenevano al medesimo Dipartimento, per gli altri, non potendo raggiungere altrimenti il numero critico (Baldry, 2005), sono stati aggregati infermieri di Dipartimenti diversi (Tabella II). Ciò, supportato dal fatto che in letteratura non c'è consenso circa l'omogeneità o eterogeneità dei componenti il gruppo (Kitzinger, 1995).

Secondo Baldry (2005), il focus group prevede la presenza di un conduttore, un osservatore e un segretario.

\begin{tabular}{|c|c|c|c|c|c|c|c|c|c|c|c|}
\hline \multirow{2}{*}{\begin{tabular}{|c|}
$\begin{array}{c}\text { Date di } \\
\text { realizzazione }\end{array}$ \\
\\
Dipartimento \\
\end{tabular}} & \multicolumn{3}{|c|}{ 22-Jun-07 } & \multicolumn{3}{|c|}{ 25-Sep-07 } & \multirow{2}{*}{\multicolumn{2}{|c|}{$\begin{array}{c}\text { 27-Sep-07 } \\
\begin{array}{c}\text { Medicine Specialità Mediche e } \\
\text { Lungodegenza }\end{array}\end{array}$}} & \multirow{2}{*}{\multicolumn{2}{|c|}{\begin{tabular}{|c|} 
9-Oct-07 \\
$\begin{array}{c}\text { Chirurgia Generale e Specialità } \\
\text { Chirurgiche }\end{array}$
\end{tabular}}} & \\
\hline & \multicolumn{2}{|c|}{$\begin{array}{c}\text { Onco- } \\
\text { Ematologico e } \\
\text { di Malattie } \\
\text { Infettive }\end{array}$} & \multirow{2}{*}{\begin{tabular}{|c|} 
Ostetrico- \\
Ginecologico- \\
Pediatrico e di \\
Neonatologia
\end{tabular}} & \multicolumn{2}{|c|}{$\begin{array}{l}\text { Cardio- } \\
\text { Pneumo e } \\
\text { di Terapia } \\
\text { Intensiva }\end{array}$} & \multirow{2}{*}{\begin{tabular}{|}
$\begin{array}{r}\text { Neuro- } \\
\text { Motorio- } \\
\text { Geriatrico }\end{array}$ \\
Cardiologia
\end{tabular}} & & & & & \\
\hline \multirow{8}{*}{$\begin{array}{c}N^{\circ} \text { INFERMIERI } \\
\text { E } \\
\text { UNITA' } \\
\text { OPERATIVE } \\
\text { DI PROVENIENZA }\end{array}$} & 1 & Bassa Carica Microbica & & 1 & & & 1 & Medicina $1^{\circ}$ & 1 & Chirurgia $1^{\circ}$ & \\
\hline & 1 & & Oncologia & 1 & \multicolumn{2}{|r|}{ Pneumologia } & 1 & Medicina $2^{\circ}$ & 1 & Chirurgia $2^{\circ}$ & \\
\hline & 1 & \multicolumn{2}{|c|}{ Malattie Infettive } & 1 & \multicolumn{2}{|c|}{ Terapia Intensiva } & 1 & Medicina $3^{\circ}$ & 1 & Room Chirurgie & \\
\hline & 1 & \multicolumn{2}{|r|}{ Ginecologia } & 1 & \multicolumn{2}{|r|}{ Neurologia } & 1 & Nefrologia & 1 & Urologia & \\
\hline & 1 & \multicolumn{2}{|r|}{ Pediatria } & 1 & \multicolumn{2}{|c|}{ Room Ortopedia } & 2 & Lungodegenza & 1 & $\begin{array}{c}\text { Chirurgia Degenza } \\
\text { Breve }\end{array}$ & \\
\hline & 1 & \multicolumn{2}{|r|}{ Neonatologia } & 1 & & Geriatria & & & \multirow{3}{*}{1} & \multirow{3}{*}{$\begin{array}{c}\text { Chirurgia Toracica, } \\
\text { Vascolare e Libera pro- } \\
\text { fessione }\end{array}$} & \\
\hline & & & & 1 & & Ortopedia & & & & & \\
\hline & & & & 1 & & $\begin{array}{l}\text { edicina Fisica } \\
\text { Riabilitativa }\end{array}$ & & & & & \\
\hline $\begin{array}{c}\text { Totale infermieri } \\
\text { identificati }\end{array}$ & \multicolumn{3}{|r|}{6} & \multicolumn{3}{|r|}{9} & \multicolumn{2}{|r|}{6} & \multicolumn{2}{|r|}{6} & 27 \\
\hline $\begin{array}{c}\text { Totale infermieri } \\
\text { effettivamente } \\
\text { presenti }\end{array}$ & \multicolumn{3}{|r|}{5} & \multicolumn{3}{|r|}{9} & \multicolumn{2}{|r|}{4} & \multicolumn{2}{|r|}{4} & 22 \\
\hline
\end{tabular}


"Quale modello teorico infermieristico, implicito o esplicito è utilizzato nella pratica assistenziale dal vostro gruppo professionale?"

Domanda 1 che cos'è un modello teorico?

Domanda 2 che cos'è un modello teorico implicito?

Domanda 3 che cos'è un modello teorico esplicito?

Domanda 4 quali caratteristiche connotano il modello teorico esplicito del gruppo professionale di appartenenza?

Tabella III. Domanda generale e domande specifiche presentate al focus

Quale conduttore è stato scelto il Referente aziendale per la Formazione, non un Tutor del Corso di Laurea perché avrebbe potuto condizionare negativamente i partecipanti, inibendo la libera espressione dei loro pensieri o portandoli ad esprimere quello che ritenevano il conduttore volesse sentirsi dire. Per ridurre il numero di persone esterne presenti, un Tutor del Corso di Laurea ha svolto sia il ruolo di osservatore che quello di segretario. In veste di osservatore, ha annotato le modalità di interazione fra $\mathrm{i}$ componenti del gruppo, la comunicazione non verbale e paraverbale, considerate indispensabili per comprendere ed interpretare meglio i contenuti verbalmente espressi (De Marinis et al., 2004b). Come segretario ha provveduto a registrare l'incontro allo scopo di ridurre i bias di analisi ed aumentare l'affidabilità del processo di elaborazione dei dati ed alla successiva trascrizione letterale di quanto registrato.

Il conduttore ha aperto, presentando la finalità dello studio, il motivo della registrazione e la durata dell'incontro, inoltre ha dato inizio al focus "lanciando" al gruppo una domanda, non specifica e molto ampia: "Quale modello teorico infermieristico, 'implicito' o 'esplicito' è utilizzato nella pratica assistenziale dal vostro gruppo professionale?". Questa domanda è stata declinata, dal conduttore, in domande più specifiche allo scopo di dettagliare meglio ed in modo progressivo il tema in oggetto, non tralasciandone aspetti importanti (Tabella III).

Il conduttore ha agito anche da: attivatore della discussione/confronto, facilitatore delle interazioni fra i componenti, incoraggiando la partecipazione di tutti e riconducendo la discussione entro gli obiettivi del focus (De Marinis et al., 2004b).

\section{ANALISI DEI DATI}

I dati raccolti sono stati analizzati secondo la tecnica di analisi del contenuto, che prevede cinque fasi fondamentali: immersione nei dati (familiarization), identificazione di una cornice tematica (identifying a thematic frame work), indicizzazione o codifica (idexing), costruzione delle tabelle tematiche, categorie e sottocategorie (charting), mappatura ed interpretazione dei dati (mapping and interpretation) (De Marinis et al., 2004b). Il segretario ha provveduto ad associare i conte- nuti alle categorie individuate (Tabella IV) in modo mutualmente escludente.

Allo scopo di verificarne la congruenza, le categorie ed i contenuti ad esse associati sono stati riletti dal conduttore e da un altro Tutor del Corso di Laurea, al di fuori dello studio e senza familiarità con i contenuti, ma con esperienza nell'analisi di focus groups.

\section{RISULTATI}

Sono stati effettuati tutti i focus calendarizzati, ma a causa di problemi derivanti dalla copertura dei turni ospedalieri, hanno partecipato 22 infermieri dei 27 inizialmente identificati (Tabella II).

In Tabella IV, sono riportate esemplificazioni dei contenuti emersi nei focus, raggruppati in categorie e sottocategorie.

Nella categoria Modello teorico sono comprese tutte le espressioni che i professionisti hanno utilizzato per descriverne il significato.

Il modello teorico, in quanto strada da seguire, traccia, immagine mentale, è un riferimento che orienta i comportamenti e che motiva ad agire. Il modello teorico è considerato un riferimento interno, personale influenzato dai propri principi, valori, esperienze aspettative legate al ruolo, ma anche qualcosa che può derivare dal confronto con altri.

Nella categoria Modello teorico implicito sono inserite tutte le modalità con le quali i professionisti descrivono il modello mentale personale che tacitamente orienta l'agire professionale relativamente al livello di consapevolezza e a come emerge o viene acquisito/trasmesso all'interno di un gruppo professionale.

Implicito perché è un modello teorico non dichiarato, "sottinteso", frutto dell'esperienza di ciascun professionista e che viene agito senza pensare. Il modello teorico implicito è quello che, all'interno del gruppo, tutti, in gran parte, utilizzano. Non è insegnato, ma viene appreso per imitazione e cambia se cambiano gruppo, contesto o condizioni organizzative.

Nella categoria Modello teorico esplicito sono racchiuse le espressioni attraverso le quali i professionisti descrivono la struttura teorica che consapevolmente, razionalmente ed intenzionalmente guida la loro pratica quotidiana dettagliandone $\mathrm{i}$ vantaggi per $\mathrm{i}$ professionisti e per il paziente stesso. 
"Quale modello teorico implicito o esplicito è utilizzato nella pratica assistenziale dal vostro gruppo professionale?"

\section{A. MODELLO TEORICO}

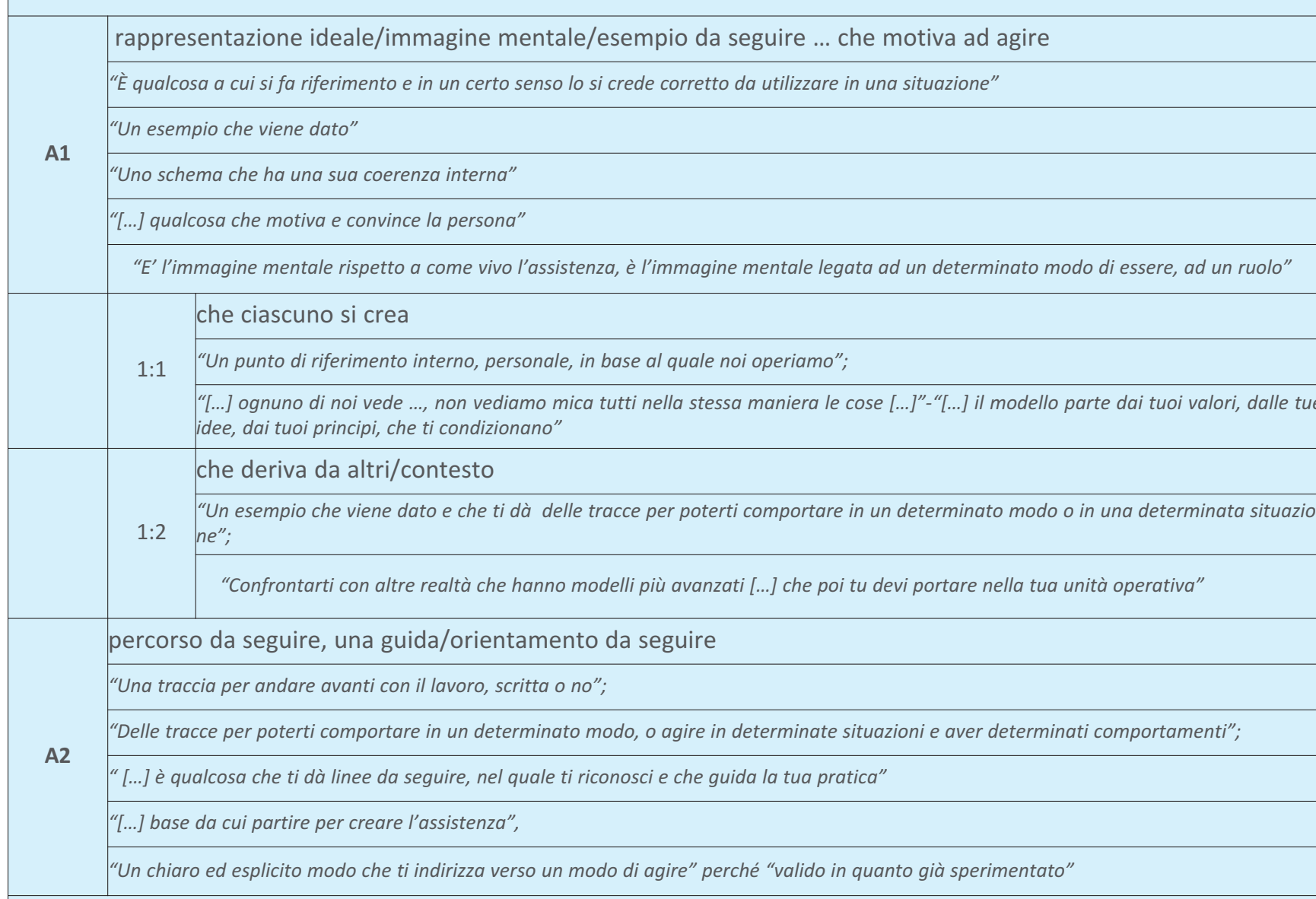

\section{B. MODELLO TEORICO IMPLICITO}

\begin{tabular}{|c|c|}
\hline \multirow{11}{*}{ B.1 } & livello di consapevolezza personale \\
\hline & "Qualcosa che è dentro e non c'è bisogno di specificarlo"; \\
\hline & "Qualcosa [...] che c'èe, che è forse più importante dell'esplicito, che coinvolge la tua esperienza, il tuo vissuto"; \\
\hline & " [...] il mio modo di lavorare è dato da come sono, dalla mia esperienza più personale"; \\
\hline & "Schema mentale che rispecchia e soddisfa la mia persona"; \\
\hline & "Testimonianza di un modo di essere"; \\
\hline & "Bisogna fare attenzione (aver chiaro) che modello implicito è per me, [...] ma può non essere valido per altri individui"; \\
\hline & "[...] è un modello che ho io come professionista ma che non lo condivido in un gruppo" \\
\hline & "La ragazzina che guarda la tv è un modello implicito quando non si rende conto che vuol diventare così, implicito ti arriva il bombardamen- \\
\hline & "Implicito ce l'ho e non me ne rendo neanche conto"; \\
\hline & "Implicito lo faccio naturalmente (senza pensare)" \\
\hline \multirow{6}{*}{ B.2 } & come emerge o viene acquisito/trasmesso all'interno di un gruppo professionale \\
\hline & "In un gruppo di lavoro, è quello che tutti conoscono e tutti usano [...] è quello prevalentemente utilizzato"; \\
\hline & "Una serie di azioni che facciamo che sono dettati da quel contesto [...] da quella specifica organizzazione che richiede quella cosa li, in que \\
\hline & "In un modello implicito, non c'è niente di scritto. Si fa così, perché ce lo si è tramandato"; \\
\hline & "Ciò che è implicito in un determinato contesto può non esserlo in un altro o in un tempo diverso". \\
\hline & $\begin{array}{l}\text { "Implicito è una cosa che è sottintesa, e quindi sarà, penso, qualcosa che tu devi arrivare a capire, che però non ti è mostrato chiaramente e } \\
\text { sei tu che devi cercare di scoprire, cioè andare oltre, leggere tra le righe" }\end{array}$ \\
\hline
\end{tabular}

Tabella IV. Tematiche principali e categorie emerse dall'analisi del contenuto dei focus group (Categorie A - B) 


\section{MODELLO TEORICO ESPLICITO}

\begin{tabular}{|c|c|}
\hline \multirow{4}{*}{ C.1 } & livello di consapevolezza personale \\
\hline & "E’ un filo che unisce, ma al tempo stesso flessibile, dunque tale da lasciare spazi di personalizzazione"; \\
\hline & $\begin{array}{l}\text { "E'quel filo che ci unisce nei nostri comportamenti principali sul lavoro, in modo che tutti cerchiamo di soddisfare i bisogni del paziente nello ste } \\
\text { so modo, perché nel momento in cui uno fa in un modo e l'altro fa in un altro, si genera il caos, cioè non c'è più assistenza"; }\end{array}$ \\
\hline & $\begin{array}{l}\text { "E' vero che ognuno di noi dà la propria, cioè come posso dire, lo può perfezionare, può dare delle interpretazioni sue personali, (ricollegate u } \\
\text { po' a tante cose nostre come può essere la nostra esperienza le nostre capacità), comunque di base rimane il modello, a partire dal quale si lava }\end{array}$ \\
\hline & vantaggi dell'esplicitazione del modello teorico \\
\hline
\end{tabular}

"Non tutti agiamo nella stessa maniera ma perseguiamo gli stessi obiettivi, ogni persona è a sé, sarebbe brutto lavorare tutti nella stessa maniera, ognuno di noi si deve distinguere come persona. Tutti comunque alla fine dobbiamo raggiungere lo stesso obtv, non è che ognuno faccia quello che gli pare";

"Rendere esplicito un modello può aiutare a pensare anche che forse sbaglio, può aiutare la riflessione e può permettere di cambia re modello. [...]";

$1: 1$

"Esplicitare il modello serve anche a evitare frustrazioni professionali. Se esplicito il modello cerco di renderlo il più possibile realiz zabile";

"Esplicitare un modello significa documentare, anche perché la gente cambia e avere qualcosa di scritto ti dà delle ideologie sempre uguali, sempre quelle";

'Cerca di uniformare un po' quella che è l'assistenza perché se no ognuno ne dà la propria interpretazione e si lavora un po' così a casaccio";

"Linea da seguire perché almeno tutti possano avere un modo di lavorare abbastanza uniforme, anche perché poi sono diverse le figure con le quali ti rapporti, con diverse capacità con diversi anni di servizio, quindi almeno per avere una linea piuttosto comune",

"Visibile, osservabile ed oggettivabile da parte anche di altri ed anche valutabile"; Anche perché se non espliciti il modello non so neanche cosa andare a cercare poi [...]. Non ho nessun obiettivo da valutare se non ho un modello dietro che mi dice. [...]"

\section{come è espresso dal gruppo professionale}

"il modello esplicito è ciò che vedo, lo trovo scritto e non ho bisogno di impegnarmi a capire o di andare oltre al suo significato, cioè lo vedo, è li, è questo e lo seguo, [...] una collega che vedo, osservo lavorare ... questo è per me un modello esplicito;

"Documentato, scritto";

"Esplicito ciò che si traduce in azione, cioè lo vedo. Il ragionamento, un pensiero viene tradotto in azione";

C.3 "La cartella [infermieristica] probabilmente è un modello esplicito. Uno dall'esterno viene noi gli possiamo anche dire che condividiamo un altro modello, ma lui dalla cartella vede che ci sono dei dati ... ed allora lui può dire 'a me non sembra, perché ciò che viene esplicitato è un'altra cosa!'”;

"Come faccio a capire il modello esplicito? Vedendo come lavorano i colleghi. domandando ai colleghi, leggendo la cartella inf., il piano di lavoro. Se è scritto è più semplice"

"Assumere un atteggiamento personale però facendo riferimento a uno standard di gruppo, un modello comune"

"Il gruppo definisce delle priorità rispetto a delle altre. E per il gruppo agire significa tenere conto di quelle priorità. Perciò tra colleghi so che agiamo nella stessa maniera. Linea comune"

Come è influenzato dalla tipologia di paziente

"Dipende anche dalla specificità del malato, perché il malato della penumo ha esigenze diverse da quello ortopedico, geriatrico ....";

"Sulla persona cardiopatica, broncopneumopatica, ultraottantenne, arrivato da qualsiasi reparto, allettato .... Noi dobbiamo lavorare e fare in modo che recuperi al meglio quelle attività di vita quotidiana che svolgeva prima";

"Pensando alla mia unità operativa, il modello ci consente di definire i bisogni peculiari dei pazienti in carico differenziandoli dai bisogni de pazienti in carico ad un'altra Unità operativa"

Tabella IV. Tematiche principali e categorie emerse dall'analisi del contenuto dei focus group (Categoria C) 


\section{MODELLO TEORICO ESPLICITO DEL RISPETTIVO GRUPPO PROFESSIONALE DI APPARTENENZA}

orientato ai bisogni della persona

"lo ti direi la Henderson, perché prendiamo come riferimento i bisogni, però credo che sia una deviazione mentale anche nel senso che quando ho fatto la scuola io come teoriche di riferimento e come modelli non ne venivano proposti altri";

"I bisogni da indagare o da soddisfare o da valutare, perché forse non siamo tutti uguali lì ....",

D.1

"Modello esplicito di riferimento è quello della Henderson, ma non so se riesco a dimostrartelo dalla mia cartella";

“La nostra cartella infermieristica è divisa per bisogni, per obiettivi con una valutazione settimanale ed un'anamnesi all'inizio che è al di là dei biso gni della Henderson (libertà dal dolore ...). Questo è il modello esplicito in realtà non viene utilizzato da tutti anche se tutti l'abbiamo condiviso, perché si fa fatica, perché c'è poco tempo, perché non c’è in tutti la cultura di usarlo perché qualcuno è più comodo lavorare per compiti ...";

"Nel mio reparto facciamo riferimento al modello che considera la persona portatrice di bisogni che vanno soddisfatti".

ai bisogni bio-fisiologici

1:1 "[...] dall'ausiliario, all'OSS, all'infermiere, al medico lavoriamo tutti insieme per il bisogno di alimentazione, di eliminazione, di mobilizzazio ne, igiene, .... ma proprio tutti insieme nel senso che non è che l'OSS pensa solo all'alimentazione ed all'igiene ed io penso solo alla mobiliz zazione e all'evacuazione, ma lavoriamo proprio ... ci coordiniamo insieme"

ai bisogni psico-emotivi e socio-culturali

"Noi facciamo spesso dimissioni protette, quindi abbiamo stretti contatti con l'assistente sociale, uno stretto contatto con il parente affin

$1: 2$ ché quando il paziente va a casa abbia un'assistenza adeguata";

"Se penso ai nostri, visto che stanno ricoverati parecchio, sicuramente, infatti vediamo che dalla nostra cartella infermieristica occupa un gran spazio, il bisogno di sicurezza e di comunicazione"

bisogni specifici di contesto

"Probabilmente il modello condiviso da tutti è quello dei bisogni di assistenza, ma è chiaro che prevalgono quello cardio-circolatorio e respi ratorio perché purtroppo sono in una situazione di emergenza";

$1: 3$

"[...] risposta ai bisogni del malato, che sono differenti, secondo me, alcuni sono di base, però ci sono alcune unità operative dove emergo no bisogni diversi più importanti di altri: un paziente che è in rianimazione e che è in coma, io penso che forse in quel momento ha bisogno di qualcosa di diverso che può essere l'accudimento"

orientato alla patologia, ai trattamenti e all'esecuzione delle tecniche

"Talvolta la tecnica sovrasta ... il punto di vista dal quale guardare la persona che si sta assistendo";

"Se il paziente, uscito dalla Sala operatoria mi chiede ho sete ed il suo bisogno è soddisfare la sua sete, però per me non può bere, perché ci sono delle priorità che devo seguire rispetto al suo bisogno, il suo bisogno (sembra cattivo dirlo!) .... come lui lo percepisce....... viene messo da parte ............ "C'è un modello che io non condivido, che, però adesso ha preso piede ed è quello di trattare i malati in orizzontale anziché in verticale. Su 11 post letto in terapia intensiva io vorrei lavorare in verticale, ossia vorrei andare dal mio malato con la mia grafica, guardare che cos'ha in corso, rilevare i parametri, la diuresi, la temperatura, ossia lavorare per quel malato ... adesso invece abbiamo un sistema di monitoraggio/rilevazione parametr vitali tutto computerizzato per cui c'è quello che parte modello facchino tac e tac e tac a tutti i malati la pressione .... per compiti... siamo tornati ad un modello organizzativo per compiti..."

"Ti spiego quale è la mia realtà, io lavoro in cardiologia, con malati in emergenza, con questo modello hub and spoke per cui ti sparano il malato da Guastalla a Reggio, sta lì se sei fortunato 24 ore e poi te lo sparano via. lo questi bisogni neanche li immagino, ma ti invidio ... ti giuro...., mi piace rebbe ...... A noi arrivano che hanno un infarto e loro ti dicono avrei "la cacca" ... e tu gli dici "no!, non la puoi fare" a differenza della sua situazio ne noi non riusciamo a farci carico del paziente e seguirlo nei suoi bisogni [...]"

Tabella IV. Tematiche principali e categorie emerse dall'analisi del contenuto dei focus group (Categoria D)

Modello teorico esplicito perché è un riferimento reso visibile dall'identificazione delle priorità assistenziali, dalla definizione degli obiettivi per il paziente e dalla scelta degli strumenti informativi. Esso dichiara, anche agli altri membri dell'equipe professionale, lo specifico ambito di competenza dell'infermiere, contribuisce a dare evidenza ai risultati raggiunti e rappresenta una guida per la riflessione sulla pratica quotidiana e dunque per processi di valutazione. Proprio perché esplicito, nell'ambito dell'equipe professionale, garantisce l'uniformità e la condivisione di comporta- menti, la continuità assistenziale e la personalizzazione.

La categoria Modello teorico esplicito del rispettivo gruppo professionale di appartenenza contiene significati relativi al modello teorico effettivo, reale, così come è agito sul paziente. Gli infermieri lo identificano con termini che richiamano reminiscenze scolastiche, quali, per esempio, il riferimento a Virginia Henderson ed a ciò che caratterizza il modello teorico dell'Autrice (bisogni della persona, soddisfazione dei bisogni, dimensione del bisogno, bisogni di base, indipendenza...). 
Gli infermieri, riferendosi al modello teorico del proprio gruppo professionale, riconoscono che si tratta di un adattamento di quanto appreso a "scuola", giacchè:

- la cartella infermieristica non è coerente con il modello dichiarato;

- si lavora per compiti;

- prevalente è l'orientamento ai bisogni bio-fisiologici o comunque ai bisogni connessi alla patologia ed ai trattamenti;

- manca una visione olistica della persona;

ma anche perché, nell'applicazione pratica del modello, il professionista si rende conto che, le peculiarità assistenziali dei pazienti in carico, non riescono ad essere esaustivamente rappresentate dai bisogni di Henderson.

\section{DISCUSSIONE}

Da quanto esitato dai focus, è possibile sostenere che gli infermieri hanno chiaro il significato di modello teorico infermieristico, riconoscendo in maniera pressoché uniforme che si tratta di un riferimento la cui finalità è quella di orientare il modo di agire l'assistenza

Ogni infermiere possiede immagini mentali delle quali può o meno esserne consapevole, che motivano e sostanziano ogni azione e scelta professionale. Si tratta di modelli interpretativi costruiti sulla base, da un lato, dell'esperienza accumulata, dall'altro, delle norme, dei valori morali ed etico-deontologici che orientano l'agire professionale ed è attraverso tale "filtro" che il professionista seleziona una porzione di ambiente rilevante ed attribuisce significato ai fatti. Il modello teorico implicito si sviluppa e rafforza nel compimento delle attività lavorative, non deriva dall'apprendimento consapevole di contenuti teorici, non è solitamente sottoposto a riflessione, verifica o condivisione, non riconosce intesa esplicitata nell'ambito del gruppo professionale.

L'esplicitazione di tali personali costrutti, non genera il modello teorico esplicito. Quest'ultimo è, infatti, il risultato di un processo sistematico e condiviso di elaborazione teorica da parte di un gruppo professionale finalizzato secondo Fawcett (2013) a fornire un razionale a ciò che gli infermieri fanno e rendere manifeste le conoscenze distintive che vengono agite nella pratica quotidiana. In maniera coerente gli infermieri riconoscono che l'esplicitazione del modello teorico consente:

- condivisione di obiettivi assistenziali per il paziente "non tutti siamo uguali e neppure i pazienti lo sono";

- uniformità delle modalità di presa in carico della persona "anche perché diverse sono le figure con le quali ti rapporti, con diverse capacità, con diversi anni di servizio";

- visibilità, anche per altri professionisti, degli esiti conseguiti sui pazienti e loro valutazione "visibile, osservabile ed oggettivabile da parte anche di altri ed anche valutabile";

- riflessione sui propri personali riferimenti impliciti e/o razionale alla base del proprio agire "esplicitare il modello può aiutare la riflessione";

- possibilità di documentare gli interventi realizzati avvalendosi di strumenti informativi condivisi.

La letteratura (Degan, 2001) riporta che i modelli teorici, benché abbiano avuto il grande merito di aver incominciato a rendere gli infermieri italiani consapevoli di quale è il loro specifico professionale e dunque del contributo originale che è loro assegnato nell'ambito della salute/malattia, non sono riusciti, di fatto, a trovare una corrispondente traduzione nella pratica quotidiana definendo modalità e strumenti per l'infermieristica clinica. Gli stessi infermieri confermano ciò nella misura in cui segnalano che dalla cartella infermieristica non emerge il riferimento teorico o che ci si "scontra" con un orientamento prioritario alla patologia, ai trattamenti e/o alle tecniche ed una conseguente frammentazione della persona.

A sostegno degli Autori (Im et al., 1999; Degan, 2001; Marrs et al., 2006) che prospettano il superamento del 'mito della grande teoria unificatrice' ed aprono alla possibilità di illuminare l'agire assistenziale attraverso teorie elaborate dalla pratica stessa, il fatto che il modello teorico esplicito del gruppo professionale non è contrapposto ai contenuti appresi, ma costruito attraverso esperienze condivise e alla capacità di "teorizzare" - ossia di riflettere - attorno alla propria prassi. La specifica peculiarità dell'agire pratico, infatti, porta il professionista ad accrescere il sapere disciplinare attraverso la re-interpretazione ed integrazione di diversi modelli, derivanti dalle precedenti esperienze, con le informazioni connesse alla specifica situazione assistenziale. La contestualizzazione del modello potrebbe non essere completamente congruente con il modello teorico di riferimento originale, proprio perché si tratta di una applicazione riflettuta "surrogata” (Wimpenny, 2002) del modello teorico.

\section{CONCLUSIONI}

Lo studio non ha inteso risolvere l'annoso problema dell'applicabilità dei modelli teorici nella pratica assistenziale, piuttosto evidenziare come il sapere teorico trovi una specifica/coerente applicazione nell'ambito della pratica.

Ragionare sull'uso della teoria nella pratica ha permesso, attraverso l'esplicitazione di conoscenze tacite 
e la condivisione del sapere esplicito, di dare visibilità a un pensiero autenticamente Infermieristico nei diversi gruppi professionali. I contributi portati hanno consentito di riconoscere e condividere la complessità del bisogno di assistenza: oggetto della disciplina Infermieristica.

L'identificazione di un campione non probabilistico, attraverso il coinvolgimento di Infermieri Guida di tirocinio, ritenuti più sensibili all'argomento perché più avvezzi al linguaggio utilizzato, rende i risultati della ricerca esplorativi del tema in oggetto.

Questa indagine rappresenta però una prima esperienza nel panorama italiano. Lo studio potrebbe essere riproposto con un campione più rappresentativo affinchè i risultati siano generalizzabili.

A livello locale, l'esplicitazione del modello teorico di riferimento emerso dai focus ha permesso di:

- avviare un processo di revisione/aggiornamento, condiviso con l'Organizzazione, del modello teorico proposto nelle docenze, nei laboratori e nei tirocini del Corso Laurea di Infermieristica;

- iniziare ad utilizzare, in alcune Unità Operative, un linguaggio disciplinare specifico (tassonomiaIm et al., ) nella documentazione del processo assistenziale per la presa in carico della persona.

E' con la promozione di processi di condivisione, di pratiche riflessive sull'esperienza e di traduzione dei saperi impliciti in concrete pratiche operative, che il modello teorico esplicito del gruppo professionale può andare incontro ad ulteriori processi di concettualizzazione, tali da arricchirlo di significati (Stephen et al., 2010).

Chiarire il proprio punto di vista, ossia la propria cornice di riferimento (Popper, 1995; Manara, 2002a), la propria e particolare visione sulle "modalità di accadere" dell'evento assistenza, rendere il più possibile esplicito il proprio agire, contribuisce a costruire teoria dalla pratica. Ogni "teoria" origina da una visione peculiare della "prassi" e, viceversa, ogni "prassi" riconosce, ex ante o ex post, una "teoria" più o meno "scientifica", più o meno motivata eticamente o culturalmente, più o meno consapevole. Diviene perciò fondamentale continuare a stressare il concetto di unità fra teoria e pratica la cui relazione di reciprocità è evidenziata dal fatto che le Teorie crescono e si sviluppano dalla Pratica, ritornano alla Pratica per essere testate e per costituire la struttura concettuale che guida l'assistenza alla persona e dà forma all'azione del prendersi cura (Mortari, 2007).

\section{BIBLIOGRAFIA}

Aggleton, P., \& Chalmers, H. (1987). Models of nursing, nursing practice and nurse education. Journal of Advanced Nursing, 12 (5), 573-81.
Alborghetti, A. (1997). L'importanza di un modello concettuale per l'assistenza infermieristica. Nursing Oggi, 3, 16-22.

Baldry, A. C. (2005). Focus group in azione. Lutilizzo in campo educativo e psicosociale. Roma: Carocci Faber.

Butts, B. J., Rich, K. L., Fawcett, J. (2012). The Future of nursing: How important is discipline -specific knowledge? A conversation with Jacquiline Fawcett. Nursing Science Quarterly, 25 (2), 151-154.

Calamandrei C. (2003). Riflessioni sull'infermieristica e sulla scienza infermieristica. Nursing Oggi, 3 (8): 16-24.

Cody, W. K. (1994). Nursing Theory-guided practice: What it is and What it is not. Nursing Science Quaterly, 7, 144-145.

Daly, J., \& Jackson, D. (1999). On the use of nursing theory in nurse education, nursing practice and nursing research in Australia. Nursing Science Quarterly, 12 (4), 342-345.

Degan, M. (2001). La nuova frontiera della produzione scientifica infermieristica: le Teorie a Medio Raggio (Middle Range Theory). Nursing Oggi, 1, 16-20.

De Marinis, M. G., Pascarella, M. C., \& Binetti, P. (2004a). Teoria e Pratica: le ragioni di un dialogo. International Nursing Perspectives, 4 (2), 87-94.

De Marinis, M. G., Piredda, M., Tartaglini, D., \& Pascarella, M. C. (2004b). Il focus group: una tecnica per la ricerca qualitativa. International Nursing Perspectives, 4 (3), 123-131.

Fawcett, J. (1992). Conceptual models and nursing practice: the reciprocal relationship. Journal of Advanced Nursing, 17 (2), 224-228.

Fawcett, J. (1999). The State of Nursing Science: Hallmarks of the 20th and 21st Centuries. Nursing Science Quarterly, 12 (4), 311-318.

Fawcett, J. (2013). Thoughts about nursing concepts. Nursing Science Quarterly, 26 (3), 285-8.

Fealy, G. M. (1999). The theory-practice relationship in nursing: the practitioners' perspective. Journal Advanced Nursing, 30 (1), 74-82.

Im, E. O., Meleis, A. I. (1999). Situation-Specific Theories: Philosophical Roots, Properties, and Approach. Advances in Nursing Science, 22 (2), 11-24.

Kenny, T. (1993). Nursing Models fail in practice. British Journal of Nursing, 2, 133-136.

Kitzinger, J. (1995). Qualitative research: Introducing focus group. British Medical of Journal, 311, 299-311.

Luker, K. (1988). Do models work? Nursing Times, 88 (5), 27-29.

Manara, D. F. (2002a). Il conflitto tra teoria e prassi nell'assistenza infermieristica. L'assistenza come saggezza pratica. Nursing oggi, 3, 16-28.

Manara, D. F. (2002b). Il conflitto tra teoria e prassi nell'assistenza infermieristica. L'assistenza come saggezza pratica. Nursing oggi, 4, 18-27.

Marrs, J. A., \& Lowry, L. W. (2006). Nursing theory and practice: connecting the dots. Nursing Science of Quarterly, 19 (1), 44-50. 
McCrae, N. (2012). Whither Nursing Models? The value of nursing theory in the context of evidence-based practice and multidisciplinary health care. Journal of Advanced Nursing, 68 (1), 222-229.

Mortari, L. (2007). Apprendere dall'esperienza. Roma: Carocci editore.

Motta, P. C. (2002). Introduzione alle scienze infermieristiche. Roma: Carocci Faber.

Popper, K. R. (1995). Il mito della cornice. Bologna: Il Mulino.

Reed, P. G., \& Lawrence, L. A. (2008). A paradigm for the production of practice-based knowledge. Journal of Nursing Management, 16 (4), 422-432.
Reed, P. G. (2008). Practitioner as Theorist. Nursing Science Quarterly, 21 (4), 315-321.

Reilly, D. (1975). Why a conceptual frame work? Nursing Outlook, 23, 566-569.

Stephen, G., \& Henry, M. D. (2010). Polanyi's tacit knowing and the relevance of epistemology to clinical medicine. Journal of evaluation in clinical practice, 16 (2), 292-297.

Tierney, A. J. (1998). Nursing Model extant or extinct? Journal of Advanced Nursing, 28 (1), 77-85.

Wimpenny, P. (2002). The meaning of models of nursing to practising nurses. Journal of Advanced Nursing, 40 (3), 346-54 